\title{
DIVERSIDADE FÍSICO-QUÍMICA DOS FRUTOS DE JABUTICABEIRAS EM UM SÍTIO DE OCORRÊNCIA NATURAL ${ }^{1}$
}

\author{
LUCAS ZERBIELLI ${ }^{2}$, ALEXANDRE AUGUSTO NIENOW ${ }^{3}$ LUANA DALACORTE $^{4}$, \\ RONALDO JACOBS ${ }^{5}$, TÁLISSON DARONCH ${ }^{6}$
}

RESUMO - Conhecer a variabilidade genética de uma espécie, manifestada em caracteres morfológicos e agronômicos, é fundamental para orientar sua conservação e manejo, e subsidiar programas de melhoramento. O objetivo deste estudo foi avaliar a diversidade físico-química dos frutos de jabuticabeiras (Plinia cauliflora) em um sítio de ocorrência natural, no município de Passo Fundo-RS. Em uma população de aproximadamente 300 jabuticabeiras, coletaram-se frutos de 40 genótipos para a avaliação de doze caracteres. Os dados foram submetidos à análise descritiva, de correlação e multivariada. Utilizou-se do método de agrupamento UPGMA, a partir da distância euclidiana média. Os genótipos G18 e G35 destacaram-se pelas características relacionadas com o tamanho e o sabor dos frutos, e também por apresentarem alta divergência genética com os demais genótipos. A relação SST/ATT foi o caractere que mais contribuiu para a divergência genética $(41,56 \%)$, seguido pela porcentagem de polpa $(25,76 \%)$ e de casca $(23,24 \%)$. Houve a formação de seis grupos de genótipos similares. Verificou-se, portanto, que jabuticabeiras nativas de um mesmo sítio de ocorrência apresentam frutos com características físico-químicas variáveis, revelando a existência de genótipos com caracteres de interesse no melhoramento da espécie.

Termos para indexação: divergência genética, frutas nativas, jabuticaba, Plinia cauliflora.

\section{PHYSICOCHEMICAL DIVERSITY OF JABUTICABA FRUITS ON A SITE OF NATURAL OCCURRENCE}

\begin{abstract}
Knowing genetic variability of species, expressed by morphological and agronomic characteristics, is essential for guiding its conservation and management to support breeding programs. The aim of this study was to evaluate the physicochemical diversity of jabuticaba fruits (Plinia cauliflora) on a site of natural occurrence in Passo Fundo, RS. In a population of approximately 300 jabuticaba trees it was collected fruits from 40 genotypes to evaluate twelve characteristics. Data were submitted to descriptive analysis, correlation and multivariate analysis. It was used UPGMA clustering method, using average Euclidean distance. The G18 and G35 genotypes distinguished by the characteristics related to fruit size and flavor and because of their high genetic divergence. The TSS/TA ratio was the characteristics which most contributed to genetic divergence (41.56\%), followed by pulp percentage $(25.76 \%)$ and peel $(23.24 \%)$. Six groups of similar genotypes were formed. It was found that native jabuticaba trees from the same site of occurrence have fruits with variables physicochemical characteristics, revealing the existence of genotypes with interesting characteristics to breeding programs of this specie.
\end{abstract}

Index terms: genetic divergence, native fruits, jabuticaba tree, Plinia cauliflora.

\footnotetext{
${ }^{1}$ Trabalho 267-14). Recebido em: 23-09-2014. Aceito para publicação em: 05-01-2015.Parte da dissertação de Mestrado em Agronomia do primeiro autor.

${ }^{2}$ Eng.-Agr., Mestre em Agronomia pelo Programa de Pós-Graduação em Agronomia (PPGAgro) da Faculdade de Agronomia e Medicina Veterinária (FAMV) da Universidade de Passo Fundo (UPF). Bolsista Capes/Fapergs. E-mail: lucaszerbi@yahoo.com.br. ${ }^{3}$ Eng.-Agr., Dr., professor do Curso de Agronomia da FAMV/UPF e do PPGAgro/UPF. E-mail: alexandre@upf.br.

${ }^{4}$ Acadêmica do Curso de Agronomia da FAMV/UPF, bolsista Pibic CNPq. E-mail: luanadalacorte@hotmail.com. ${ }^{5}$ Eng.-Agr. formado pelo Curso de Agronomia da FAMV/UPF, bolsista Probic Fapergs. E-mail: 106648@upf.br.

${ }^{6}$ Eng.-Agr. formado pelo Curso de Agronomia da FAMV/UPF, bolsista Pibic CNPq. E-mail: 99260@upf.br.
} 


\section{INTRODUÇÃO}

A jabuticabeira (Plinia spp.), frutífera pertencente à família Myrtaceae, é nativa do Brasil, e sua ocorrência é mais comum no Bioma Mata Atlântica, desde o Nordeste do País, em locais com temperaturas não muito elevadas, até o Noroeste do Planalto Médio do Rio Grande do Sul. Também pode ser encontrada e cultivada com êxito em outras regiões do estado gaúcho (MATTOS, 1983).

As jabuticabeiras produzem frutos com reconhecido potencial econômico, devido às características organolépticas. São muito apreciados para o consumo in natura e valorizados para a fabricação de derivados, como geleias, bebidas fermentadas, vinagre e licores (DANNER et al., 2006). Além disso, os frutos são ricos em compostos com funções antioxidantes e outros componentes de interesse na indústria farmacêutica (CITADIN et al., 2010). Danner et al. (2011) verificaram a presença de altos teores de antocianinas e flavonoides

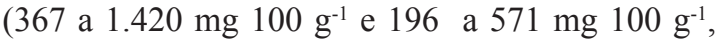
respectivamente) na casca de jabuticabas de 36 plantas nativas da região sudoeste do Paraná. O efeito positivo das antocianinas e flavonoides da casca da jabuticaba no combate a radicais livres e consequente redução de câncer de próstata, de leucemia (LEITELEGATTI et al., 2012), do colesterol e do diabetes (LENQUISTE et al., 2012) foi comprovado em testes com ratos.

No entanto, a escassez de conhecimento sobre as espécies de jabuticabeira, e o consequente baixo aproveitamento comercial, somado ao nível de degradação do Bioma Mata Atlântica, pela ação antrópica, tem reduzido gradativamente as plantas nativas e a diversidade genética. Bancos de germoplasma dessas espécies restringem-se a pequenas coleções de plantas em alguns órgãos de pesquisa. Portanto, a conservação in situ e ex situ, e a caracterização dos recursos genéticos existentes são fundamentais como estratégia para minorar os danos já causados e aumentar a exploração dessa frutífera em cultivos comerciais (CITADIN et al., 2010), em sistemas solteiros ou agroflorestais (SAFs), bem como constituindo-se em uma das espécies na recuperação de Áreas de Preservação Permanente (APPs) e Reserva Legal (RL).

O estudo das características morfológicas e agronômicas das plantas é importante para se conhecer a divergência genética do conjunto do germoplasma disponível, para fins de utilização em programas de melhoramento genético (ELIAS et al., 2007). Em trabalhos de avaliação de diversidade genética, os métodos preditivos têm sido utilizados sobretudo pelo fato de que, ao se basearem em diferenças morfológicas e fisiológicas dos genitores, dispensam a obtenção das combinações híbridas entre eles, o que é vantajoso, especialmente quando o número de genitores, cujas diversidades se deseja conhecer, é elevado. Entre os métodos preditivos estão aqueles que quantificam a diversidade por meio de medidas de dissimilaridade (CARVALHO et al., 2003; ELIAS et al., 2007).

Visando a avançar no conhecimento da jabuticabeira e fornecer subsídios para futuros trabalhos de melhoramento genético dessa frutífera nativa, o objetivo deste estudo foi avaliar a diversidade físico-química dos frutos de jabuticabeiras em um sítio de ocorrência natural.

\section{MATERIAL E MÉTODOS}

O estudo foi realizado em um sítio de ocorrência natural (mata nativa) de jabuticabeiras, no município de Passo Fundo-RS (latitude $28^{\circ} 13^{\prime} \mathrm{S}$; longitude $52^{\circ} 27^{\prime} \mathrm{O}$; altitude $655 \mathrm{~m}$ ), coletando-se frutos de plantas (genótipos) adultas, pertencentes à espécie Plinia cauliflora. A idade das plantas não pôde ser estabelecida, mas pelo elevado porte, provavelmente, algumas eram centenárias.

Segundo a classificação climática de Köeppen, o clima da região é do tipo $\mathrm{Cfa}$, úmido em todas as estações do ano, com chuvas bem distribuídas e verão quente (KUINCHTNER; BURIOL, 2001). De acordo com as Normais Climatológicas (1961/1990) registradas pelo Laboratório de Agrometeorologia da Embrapa Trigo de Passo Fundo-RS, a temperatura média anual do município é de $17,7^{\circ} \mathrm{C}$, com média das máximas de $23,6{ }^{\circ} \mathrm{C}$ e média das mínimas de $13,2^{\circ} \mathrm{C}$. A precipitação média anual é de $1.800 \mathrm{~mm}$ (EMBRAPA, 2013). O solo do local de estudo é classificado como Latossolo Vermelho distrófico húmico (Unidade Passo Fundo) (STRECK et al., 2008).

$\mathrm{Na}$ área, havia, aproximadamente, 300 plantas de jabuticabeira, mas no trabalho utilizouse de 40 genótipos que apresentavam produção significativa e frutos maduros na época de coleta, realizada em outubro de 2012.

Avaliaram-se as características físicoquímicas dos frutos, coletando-se amostras de 30 frutos por planta, no estádio de maturação completa (coloração preto-arroxeada), em ramos dispostos radialmente na porção mediana da copa. Após prévia lavagem em água corrente, no Laboratório de Ecofisiologia Vegetal da FAMV/UPF, avaliaramse 12 características físico-químicas dos frutos: diâmetro longitudinal (DLF) e transversal (DTF) 
(mm), utilizando-se de paquímetro digital; massa fresca dos frutos (MFF) e das sementes (MFS) (g), utilizando-se de balança analítica digital; número de sementes por fruto (NS); porcentagem de polpa, casca e semente, pesando-se cada fração individualmente; potencial hidrogeniônico $(\mathrm{pH})$, utilizando-se de pHmetro; acidez total titulável (ATT); sólidos solúveis totais (SST) $\left({ }^{\circ}\right.$ Brix), utilizando-se de refratômetro manual; e relação SST/ATT da polpa dos frutos. As análises químicas foram realizadas em triplicata de dez frutos. A ATT foi determinada por titulação com hidróxido de sódio $(\mathrm{NaOH})$ a $0,1 \mathrm{~N}$ até $\mathrm{pH} 8,1-8,2$, utilizando-se de 10 $\mathrm{g}$ de polpa diluídos em $90 \mathrm{~mL}$ de água destilada. Os valores da acidez foram expressos em porcentagem de ácido cítrico, conforme metodologia descrita pelo Instituto Adolfo Lutz (1985).

Os dados foram analisados por estatística descritiva, e as diferenças entre os genótipos estabelecidas, considerando-se um desvio-padrão em relação à média. Calculou-se o coeficiente de correlação de Pearson entre as variáveis, verificando-se a significância pelo teste $t(\mathrm{P}>0,01)$. Para estimar a divergência genética entre os genótipos, a partir das médias padronizadas das características estudadas, obteve-se a matriz de dissimilaridade, gerada com a distância euclidiana média, avaliando-se, ainda, a contribuição relativa das características físico-químicas na variabilidade observada entre os 40 genótipos, pelo método de Singh (1981). Com a matriz de dissimilaridade, foi gerado um dendrograma, utilizando o método de agrupamento Unweighted Pair Group Method using Arithmetic Averages (UPGMA), ou ligação média entre grupos. $\mathrm{O}$ ponto de corte, para a definição dos grupos no dendrograma, foi determinado pelo método subjetivo, com base no conhecimento do material estudado. Todas as análises estatísticas foram realizadas no programa computacional Genes (CRUZ, 2006).

\section{RESULTADOS E DISCUSSÃO}

A espécie de jabuticabeira encontrada no sítio de ocorrência natural estudado foi a $P$. cauliflora. Antes do início desta pesquisa, acreditava-se que, na região, a espécie nativa seria predominantemente $P$. trunciflora. Danner et al. (2010) também verificaram a ocorrência natural da espécie $P$. cauliflora, ao realizarem o mapeamento de 14 remanescentes florestais, na região sudoeste do Estado do Paraná, pertencentes ao ecossistema, Floresta Ombrófila Mista (Floresta de Araucária), Bioma Mata Atlântica. Portanto, por se tratar do mesmo ecossistema a área em que o estudo foi realizado, é possível reafirmar que $P$. cauliflora também é nativa da região do Planalto Médio do Rio Grande do Sul.

$\mathrm{O}$ diâmetro longitudinal dos frutos (DLF) variou de $21,6 \mathrm{~mm}$ a $26,0 \mathrm{~mm}$, e o transversal (DTF), de $21,7 \mathrm{~mm}$ a 26,5 mm. Frutos de maior DLF, acima de 1 desvio-padrão da média, foram produzidos pelos genótipos G18, G20, G21, G22, G25, G31 e G35, variando de 25,1 a $26,0 \mathrm{~mm}$ (Tabela 1 ). Quanto ao DTF, apenas o G21 e o G25 não se mantiveram entre os genótipos que haviam apresentado maior DLF, passando o G19 a integrar o grupo, variando de 25,4 $\mathrm{mm}$ a $26,5 \mathrm{~mm}$ (Tabela 1). O tamanho apresentado pelos frutos concorda com Mattos (1983), ao relatar que, em jabuticabeiras pertencentes à espécie $P$. cauliflora, o DLF varia entre $22,0 \mathrm{~mm}$ e $28,0 \mathrm{~mm}$; e o DTF, entre $22,0 \mathrm{~mm}$ e $29,0 \mathrm{~mm}$.

A massa fresca dos frutos (MFF) variou de 6,4 g a 11,4 g. A maioria dos genótipos que apresentaram maior DLF e DTF também produziram frutos com maior massa (G18, G19, G20, G21, G22, G25, G31 e G35), oscilando de 10,0 g a 11,4 g (Tabela 1). Citadin et al. (2005), avaliando por três anos consecutivos frutos de jabuticabeira (P. cauliflora) em condições de mata nativa, na região sudoeste do Paraná, verificaram que a massa média variou entre $8,0 \mathrm{~g}$ e $9,0 \mathrm{~g}$, concordando com a média verificada, que foi de $8,7 \mathrm{~g}$.

O número de sementes (NS) encontrado pode ser considerado baixo e pouco variável. Oscilou entre 1,0 (G8, G9, G23 e G36) e 1,7 sementes por fruto (Tabela 1). Em nenhum dos frutos analisados constatou-se a ausência de sementes. Jesus et al. (2004) obtiveram um número de sementes que oscilou entre 1,5 , próximo à média observada no presente trabalho, e 3,1, sendo esse valor considerado elevado.

A massa fresca das sementes (MFS) variou de $0,11 \mathrm{~g}$ a $0,36 \mathrm{~g}$, sendo que quatro genótipos (G13, G15, G29 e G32) apresentaram massa inferior aos demais, com apenas $0,11 \mathrm{~g}$ a $0,13 \mathrm{~g}$ (Tabela 1). Destaca-se que o genótipo G32, apesar de ter produzido frutos com sementes pequenas, foi o que apresentou maior número médio de sementes por fruto (1,7 semente).

O percentual de polpa variou de $57,8 \%$ a $76,1 \%$ da massa total do fruto; e a casca, de $20,7 \%$ a $36,5 \%$. Quando o destino dos frutos é para o consumo in natura, a porcentagem de polpa é a característica mais importante, uma vez que a casca coriácea e a(s) semente(s) são descartadas. Nesse caso, destacaramse os genótipos G8, G9, G11, G12, G16, G17 e G27, que possibilitaram a obtenção de 72,7 a $76,1 \%$ de polpa (Tabela 1). No entanto, em alguns casos, a 
casca de jabuticabas também tem grande utilidade, como na elaboração de certos derivados (bebidas fermentadas, geleias, sucos, polpa congelada, entre outros), ou para a extração de substâncias como antocianinas, para fins industriais ou farmacológicos. Portanto, a importância da porcentagem de polpa e de casca de jabuticabas fica condicionada ao destino pretendido com os frutos.

O percentual de sementes (Tabela 1), que variou de 1,9 a 5,7\%, não apresentou uma relação direta com a massa total dos frutos, ou seja, frutos maiores não rigorosamente proporcionaram menor porcentagem de sementes, sendo essa relação dependente, também, do número e do tamanho das sementes. Por exemplo, no genótipo G26, com frutos relativamente pequenos, de $8 \mathrm{~g}$, a média de 1,1 semente por fruto representou $5 \%$ da massa total, por apresentar uma semente grande $(0,36 \mathrm{~g})$; já em frutos do genótipo G3, de mesma massa fresca, com 1,2 semente, porém menores $(0,22 \mathrm{~g})$, a porcentagem representada pelas sementes foi mais baixa $(3,3 \%)$. $\mathrm{O}$ baixo número e o tamanho das sementes são características extremamente importantes para o consumo in natura, assim como para a utilização no processamento industrial, pois aumentam o rendimento.

Em relação às características da polpa dos frutos, o $\mathrm{pH}$ variou de 3,33 a 4,04, com maiores valores obtidos em oito genótipos (G3, G4, G13, G17, G18, G24, G36 e G40), entre 3,93 e 4,04 (Tabela 1). Lima et al. (2008) obtiveram variação de $\mathrm{pH}$ semelhante $(3,39$ a 4,01), ao realizarem a caracterização química de frutos inteiros de jabuticabeiras da mesma espécie, e de suas frações (polpa, casca e semente) separadamente. A acidez total titulável (ATT), de modo geral, apresentou baixas porcentagens, não ultrapassando $0,59 \%$ de ácido cítrico (Tabela 1), destacando-se um grupo de oito genótipos (G3, G4, G13, G17, G18, G35, G36 e G40) com acidez inferior aos demais, variando de 0,33 a $0,37 \%$ de ácido cítrico. Maiores porcentagens de ATT $(0,97 \%$ a $0,99 \%$ de ácido cítrico) foram obtidos por Lima et al. (2008). Em jabuticabeiras 'Sabará' (P. jaboticaba), Oliveira et al. (2003) registraram valores ainda mais elevados $(0,89$ a 1,65\% de ácido cítrico).

Quanto aos sólidos solúveis totais (SST), a variação deu-se entre $10,8^{\circ}$ Brix e $15,8^{\circ}$ Brix, sendo que 23 genótipos apresentaram teor igual ou superior à média, que foi de $13,4{ }^{\circ}$ Brix. Os maiores teores foram obtidos nos frutos dos genótipos G9, G15, G18, G19, G21 e G33 (Tabela 1), oscilando de 14,7 ${ }^{\circ}$ Brix a $15,8{ }^{\circ}$ Brix. Citadin et al. (2005) obtiveram resultados semelhantes, avaliando a qualidade de jabuticabas, por três anos consecutivos, no sudoeste do Paraná, sob mata nativa, constatando teores de SST que oscilaram entre $11,91{ }^{\circ}$ Brix e 14,51 ${ }^{\circ}$ Brix. Em frutos coletados de jabuticabeiras que frutificaram a pleno sol, os teores de SST variaram de $12,47^{\circ}$ Brix a $15,14{ }^{\circ}$ Brix.

Calculando a relação SST/ATT, oito genótipos (G3, G4, G13, G15, G17, G18, G35 e G36) sobressaíram-se em relação aos demais, com valores que variaram de 36,5 a 42,4 (Tabela 1), mas 19 genótipos posicionaram-se acima da média $(30,9)$. Danner et al. (2011) obtiveram maior variação da relação SST/ATT $(10,3$ a 63,2) em jabuticabas provenientes de 36 genótipos ( $P$. cauliflora) localizados em cinco locais (municípios) da região sudoeste do Paraná. Porém, a média verificada pelos autores $(28,5)$ ficou próxima à obtida no presente trabalho. A relação SST/ATT é um importante indicativo do sabor, pois relaciona os açúcares com os ácidos dos frutos (FACHINELLO; NACHTIGAL, 2013), determinando, pelo equilíbrio gustativo, a qualidade geral de um produto.

O cálculo da correlação de Pearson entre os doze caracteres analisados (Tabela 2) revelou a existência de variáveis altamente correlacionadas entre si. Essa correlação foi verificada entre o DLF e o DTF $(0,94)$, e dessas variáveis com a MFF $(0,93$ e 0,87 , respectivamente), assim como entre o $\mathrm{pH}$ e a relação SST/ATT $(0,86)$, e a MFS com a porcentagem de sementes $(0,81)$. Outros caracteres também tiveram correlação positiva e significativa entre si, porém representados por coeficientes menores. Algumas variáveis tiveram acentuada correlação negativa, como observado entre a porcentagem de polpa e de casca $(-0,98)$, entre pH e ATT $(-0,93)$ e entre ATT e a relação SST/ATT $(-0,87)$. A correlação negativa significa que, na medida em que se aumenta a porcentagem de polpa, decresce a porcentagem de casca, bem como a elevação do $\mathrm{pH}$ decorre da redução da ATT, e na medida em que aumenta a ATT, não havendo grandes alterações no teor de SST, a relação SST/ATT decresce.

Analisando-se a contribuição relativa dos doze caracteres avaliados (Tabela 3 ), verificou-se que a relação SST/ATT foi a que mais contribuiu para a divergência genética $(41,56 \%)$, seguida pela porcentagem de polpa $(25,76 \%)$ e de casca $(23,24 \%)$, respondendo, esses três caracteres, por aproximadamente $90 \%$ da divergência genética dos genótipos de jabuticabeira. Danner et al. (2011), ao avaliarem a divergência genética entre 36 genótipos de jabuticabeiras, com base em 16 caracteres, constataram maior contribuição da porcentagem de casca $(48,0 \%)$ e de polpa $(45,5 \%)$. Esses resultados 
confirmam a importância do percentual de polpa e de casca para a divergência genética de jabuticabeiras.

Com base nas características físico-químicas dos frutos, a medida de dissimilaridade (Tabela não apresentada), estimada pela distância euclidiana média (D), demonstrou que a maior divergência existe entre $\mathrm{G} 1$ e $\mathrm{G} 18\left(\mathrm{D}_{(\mathrm{G} 1-\mathrm{G} 18)}=2,56\right)$ e $\mathrm{G} 1$ e $\mathrm{G} 9\left(\mathrm{D}_{(\mathrm{Gl}-\mathrm{G} 9)}=2,50\right)$. As menores distâncias foram verificadas entre $\mathrm{G} 4$ e $\mathrm{G} 24\left(\mathrm{D}_{(\mathrm{G} 4-\mathrm{G} 24)}=0,33\right), \mathrm{G} 21$ e $\mathrm{G} 25\left(\mathrm{D}_{(\mathrm{G} 21-\mathrm{G} 25)}=0,33\right)$ e $\mathrm{G} 12$ e $\mathrm{G} 16\left(\mathrm{D}_{(\mathrm{G} 12-\mathrm{G} 6)}=\right.$ 0,37 ), indicando serem os genótipos mais similares. Confrontando-se todos os genótipos, constatou-se que o G2 foi o que apresentou a menor distância média em relação aos demais $(\mathrm{D}=1,05)$, e $\mathrm{G} 1$ a maior distância $(D=1,70)$, seguido por $G 32(D=1,68)$, G18 (D = 1,59), G29 (D = 1,56), G13 (D=1,53), G39 $(\mathrm{D}=1,52)$ e $\mathrm{G} 35(\mathrm{D}=1,51)$. A distância genética é maior entre plantas oriundas de diferentes sítios de ocorrência do que entre plantas localizadas no mesmo sítio, pois há maior probabilidade de se encontrar genótipos aparentados localizados próximos entre si, dispersos em pequenas distâncias. No entanto, é possível que dentro do mesmo sítio de ocorrência existam genótipos com distâncias elevadas entre si (DANNER et al., 2011).

Visando à obtenção de genótipos com características segregantes e superiores, os genótipos identificados como geneticamente mais distantes em relação aos demais poderiam ser indicados. De acordo com Cruz e Regazzi (1997), seria possível um maior efeito heterótico na geração seguinte, aumentando a probabilidade de alcançar tais características na progênie. Estudos de determinação de divergência genética entre genótipos têm sido ferramentas de grande importância em programas de melhoramento, auxiliando na identificação de genitores com potencial heterótico (VIEIRA et al., 2009).

No dendrograma (Figura 1), o ponto de corte efetuado permitiu a formação de seis grupos de genótipos similares. O Grupo 3 foi constituído por nove genótipos que, com exceção do G23 e G26, reuniu aqueles que apresentaram frutos com maior porcentagem de polpa e menor porcentagem de casca, variáveis que, juntas, tiveram grande contribuição na divergência genética (Tabela 3). Os demais grupos foram formados por 11 (Grupo 1), sete (Grupo 2), nove (Grupo 4) e três genótipos (Grupo 5), com o Grupo 6 apresentando apenas o genótipo G1. Com o dendrograma também foi possível visualizar a alocação dos genótipos conforme a divergência anteriormente discutida. Para exemplificar, os genótipos G4 e G24, G21 e G25, e G12 e G16, identificados como os pares mais similares, alocaram-se nos mesmos grupos e muito próximos, sendo que os dois primeiros pares fizeram parte do Grupo 1, e o G12 e G16, do Grupo 3. Por outro lado, os genótipos G1 e G18, e G1 e G9, identificados como os mais divergentes, constituíram grupos diferentes e visualmente muito distantes no dendrograma, sendo que o G1 ficou incluído no Grupo 6, o G18 no Grupo 1 e o G9 no Grupo 3.

O sítio de ocorrência de jabuticabeiras nativas avaliado não tem sua origem conhecida, mas considerando que nas áreas de mata nativa adjacentes não se verifica a presença dessa espécie, é de se supor que a reprodução se deu por sementes, a partir de uma ou poucas plantas. Ainda assim, confirmou-se a existência de variabilidade nas características físicoquímica dos frutos, embora nenhum genótipo tenha apresentado frutos de tamanho excepcional.

Considerando as variáveis estudadas, podem-se destacar os genótipos G18 e G35 pelas características relacionadas com o tamanho e o sabor dos frutos, e também por apresentarem alta divergência genética com os demais genótipos. Portanto, em futuros trabalhos com essa mesma população de jabuticabeiras, sugere-se que esses dois genótipos sejam alvo de maiores investigações. Além disso, conforme sugerido por

poderiam ser obtidas progênies de polinização aberta desses genótipos, para posterior avaliação das plantas em bancos ativos de germoplasma (BAGs). Da mesma forma, outros genótipos que apresentem características diferenciadas, devem ser incluídos nesses BAGs, aumentando a variabilidade genética da coleção, o que é extremamente desejável em programas de melhoramento genético. 
TABELA 1 - Características físico-químicas dos frutos de 40 genótipos de jabuticabeira (Plinia cauliflora) em um sítio de ocorrência natural. Passo Fundo, RS, 2012.

\begin{tabular}{|c|c|c|c|c|c|c|c|c|c|c|c|c|}
\hline \multirow{2}{*}{ Genótipo } & \multirow[t]{2}{*}{ DLF } & \multirow{2}{*}{$\frac{\text { DTF }}{\text { am) }}$} & \multirow{2}{*}{$\begin{array}{c}\text { MFF } \\
\text { (g) }\end{array}$} & \multirow{2}{*}{ NS } & \multirow{2}{*}{$\begin{array}{l}\text { MFS } \\
\text { (g) }\end{array}$} & \multicolumn{3}{|c|}{$\begin{array}{c}\text { Composição do fruto } \\
(\%)\end{array}$} & \multirow{2}{*}{ pH } & \multirow{2}{*}{$\begin{array}{c}\text { ATT } \\
\text { (\% ácido } \\
\text { cítrico) }\end{array}$} & \multirow{2}{*}{$\begin{array}{c}\text { SST } \\
\left({ }^{\circ} \text { Brix) }\right.\end{array}$} & \multirow{2}{*}{ SST/ATT } \\
\hline & & & & & & Po & 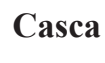 & Semente & & & & \\
\hline G1 & $22,6 \mathrm{~b}$ & $22,5 \mathrm{c}$ & $7,3 \mathrm{c}$ & $1,4 \mathrm{~b}$ & $0,30 \mathrm{a}$ & $57,8 \mathrm{c}$ & $36,5 \mathrm{a}$ & $5,7 \mathrm{a}$ & $3,74 \mathrm{~b}$ & $0,43 \mathrm{~b}$ & $11,5 \mathrm{c}$ & $27,0 \mathrm{~b}$ \\
\hline G2 & $23,2 \mathrm{~b}$ & $23,6 \mathrm{~b}$ & $7,7 \mathrm{~b}$ & $1,1 \mathrm{~b}$ & $0,21 \mathrm{~b}$ & $67,3 \mathrm{~b}$ & $29,8 \mathrm{~b}$ & $3,0 \mathrm{~b}$ & $3,72 \mathrm{~b}$ & $0,43 \mathrm{~b}$ & $13,6 \mathrm{~b}$ & $31,6 \mathrm{~b}$ \\
\hline G3 & $23,7 \mathrm{~b}$ & $23,5 \mathrm{~b}$ & $8,0 \mathrm{~b}$ & $1,2 \mathrm{~b}$ & $0,22 \mathrm{~b}$ & $64,9 \mathrm{~b}$ & $31,8 \mathrm{~b}$ & $3,3 \mathrm{~b}$ & $4,02 \mathrm{a}$ & $0,35 \mathrm{c}$ & $12,9 \mathrm{~b}$ & $36,5 \mathrm{a}$ \\
\hline G4 & $24,6 \mathrm{~b}$ & $24,8 \mathrm{~b}$ & $9,3 \mathrm{~b}$ & $1,3 \mathrm{~b}$ & $0,20 \mathrm{~b}$ & $68,6 \mathrm{~b}$ & $28,6 \mathrm{~b}$ & $2,9 \mathrm{~b}$ & $4,01 \mathrm{a}$ & $0,37 \mathrm{c}$ & $13,9 \mathrm{~b}$ & $37,8 \mathrm{a}$ \\
\hline G5 & $23,5 \mathrm{~b}$ & $23,9 \mathrm{~b}$ & 7,9 b & $1,3 \mathrm{~b}$ & $0,27 \mathrm{~b}$ & 62, & $32,9 \mathrm{~b}$ & $4,5 \mathrm{a}$ & $3,35 \mathrm{c}$ & $0,54 \mathrm{a}$ & $11,3 \mathrm{c}$ & $21,3 \mathrm{c}$ \\
\hline G6 & $23,6 \mathrm{~b}$ & $23,9 \mathrm{~b}$ & $8,2 \mathrm{~b}$ & $1,6 \mathrm{a}$ & $0,23 \mathrm{~b}$ & 64 & $31,3 \mathrm{~b}$ & $4,5 \mathrm{a}$ & $3,52 \mathrm{~b}$ & $0,48 \mathrm{~b}$ & $11,5 \mathrm{c}$ & $24,0 \mathrm{c}$ \\
\hline G7 & $24,8 \mathrm{~b}$ & $25,1 \mathrm{~b}$ & $8,4 \mathrm{~b}$ & $1,1 \mathrm{~b}$ & $0,30 \mathrm{a}$ & 67, & $29,0 \mathrm{~b}$ & $3,9 \mathrm{~b}$ & $3,35 \mathrm{c}$ & $0,52 \mathrm{a}$ & $12,2 \mathrm{~b}$ & $23,6 \mathrm{c}$ \\
\hline G8 & $24,1 \mathrm{~b}$ & $24,4 \mathrm{~b}$ & $8,7 \mathrm{~b}$ & $1,0 \mathrm{c}$ & $0,24 \mathrm{~b}$ & 72 & $24,6 \mathrm{c}$ & $2,7 \mathrm{~b}$ & $3,65 \mathrm{~b}$ & $0,45 \mathrm{~b}$ & $12,7 \mathrm{~b}$ & $28,4 \mathrm{~b}$ \\
\hline G9 & $24,4 \mathrm{~b}$ & $24,6 \mathrm{~b}$ & $9,1 \mathrm{~b}$ & $1,0 \mathrm{c}$ & $0,18 \mathrm{~b}$ & 76 , & $22,1 \mathrm{c}$ & $1,9 \mathrm{c}$ & $3,78 \mathrm{~b}$ & $0,42 \mathrm{~b}$ & $14,7 \mathrm{a}$ & $35,1 \mathrm{~b}$ \\
\hline G10 & $22,7 \mathrm{~b}$ & $23,0 \mathrm{~b}$ & $7,3 \mathrm{c}$ & $1,1 \mathrm{~b}$ & $0,27 \mathrm{~b}$ & 70 , & $26,0 \mathrm{~b}$ & $4,0 \mathrm{~b}$ & $3,39 \mathrm{c}$ & $0,53 \mathrm{a}$ & $11,3 \mathrm{c}$ & $21,2 \mathrm{c}$ \\
\hline G11 & $23,3 \mathrm{~b}$ & $23,8 \mathrm{~b}$ & $7,9 \mathrm{~b}$ & $1,2 \mathrm{~b}$ & $0,23 \mathrm{~b}$ & 73 & $22,8 \mathrm{c}$ & $3,6 \mathrm{~b}$ & $3,58 \mathrm{~b}$ & $0,47 \mathrm{~b}$ & $12,6 \mathrm{~b}$ & $26,8 \mathrm{~b}$ \\
\hline G12 & $24,9 \mathrm{~b}$ & $25,0 \mathrm{~b}$ & $9,5 \mathrm{~b}$ & $1,2 \mathrm{~b}$ & $0,23 \mathrm{~b}$ & 73 & $23,2 \mathrm{c}$ & $2,9 \mathrm{~b}$ & $3,55 \mathrm{~b}$ & $0,48 \mathrm{~b}$ & $13,0 \mathrm{~b}$ & $27,2 \mathrm{~b}$ \\
\hline G13 & $22,4 \mathrm{c}$ & $23,0 \mathrm{~b}$ & $7,5 \mathrm{~b}$ & $1,2 \mathrm{~b}$ & $0,12 \mathrm{c}$ & 66 , & $32,0 \mathrm{~b}$ & $1,9 \mathrm{c}$ & $4,04 \mathrm{a}$ & $0,33 \mathrm{c}$ & $13,6 \mathrm{~b}$ & $41,8 \mathrm{a}$ \\
\hline G14 & $24,2 \mathrm{~b}$ & $24,7 \mathrm{~b}$ & $9,0 \mathrm{~b}$ & $1,4 \mathrm{~b}$ & $0,23 \mathrm{~b}$ & 63 & $32,5 \mathrm{~b}$ & $3,7 \mathrm{~b}$ & $3,88 \mathrm{~b}$ & $0,39 \mathrm{~b}$ & $12,7 \mathrm{~b}$ & $32,4 \mathrm{~b}$ \\
\hline G15 & $22,7 \mathrm{~b}$ & $23,5 \mathrm{~b}$ & $7,8 \mathrm{~b}$ & $1,2 \mathrm{~b}$ & $0,13 \mathrm{c}$ & 64, & $33,8 \mathrm{a}$ & $2,1 \mathrm{c}$ & $3,90 \mathrm{~b}$ & $0,39 \mathrm{~b}$ & $14,9 \mathrm{a}$ & $38,4 \mathrm{a}$ \\
\hline G16 & $24,7 \mathrm{~b}$ & $25,1 \mathrm{~b}$ & $9,4 \mathrm{~b}$ & $1,1 \mathrm{~b}$ & $0,23 \mathrm{~b}$ & 75 & $21,3 \mathrm{c}$ & $2,8 \mathrm{~b}$ & $3,71 \mathrm{~b}$ & $0,45 \mathrm{~b}$ & $13,5 \mathrm{~b}$ & $30,1 \mathrm{~b}$ \\
\hline G17 & $24,1 \mathrm{~b}$ & $24,7 \mathrm{~b}$ & $9,0 \mathrm{~b}$ & $1,2 \mathrm{~b}$ & $0,24 \mathrm{~b}$ & & $20,7 \mathrm{c}$ & $3,3 \mathrm{~b}$ & $3,96 \mathrm{a}$ & $0,37 \mathrm{c}$ & $13,7 \mathrm{~b}$ & $37,6 \mathrm{a}$ \\
\hline G18 & $25,6 \mathrm{a}$ & $25,9 \mathrm{a}$ & $10,7 \mathrm{a}$ & $1,1 \mathrm{~b}$ & $0,20 \mathrm{~b}$ & & $28,6 \mathrm{~b}$ & $2,0 \mathrm{c}$ & $3,97 \mathrm{a}$ & $0,37 \mathrm{c}$ & $15,8 \mathrm{a}$ & $42,5 \mathrm{a}$ \\
\hline G19 & $24,7 \mathrm{~b}$ & $25,4 \mathrm{a}$ & $10,0 \mathrm{a}$ & $1,5 \mathrm{~b}$ & $0,20 \mathrm{~b}$ & & $25,9 \mathrm{~b}$ & $2,8 \mathrm{~b}$ & $3,49 \mathrm{~b}$ & $0,49 \mathrm{~b}$ & $15,3 \mathrm{a}$ & $31,3 \mathrm{~b}$ \\
\hline G20 & $25,1 \mathrm{a}$ & $25,7 \mathrm{a}$ & $10,2 \mathrm{a}$ & $1,5 \mathrm{~b}$ & $0,16 \mathrm{~b}$ & & $26,7 \mathrm{~b}$ & $2,4 \mathrm{c}$ & $3,48 \mathrm{~b}$ & $0,49 \mathrm{~b}$ & $13,3 \mathrm{~b}$ & $27,2 \mathrm{~b}$ \\
\hline G21 & $25,5 \mathrm{a}$ & $24,4 \mathrm{~b}$ & $11,0 \mathrm{a}$ & $1,5 \mathrm{~b}$ & $0,20 \mathrm{~b}$ & & $25,1 \mathrm{~b}$ & $2,8 \mathrm{~b}$ & $3,74 \mathrm{~b}$ & $0,43 \mathrm{~b}$ & $15,2 \mathrm{a}$ & $35,7 \mathrm{~b}$ \\
\hline G22 & $25,7 \mathrm{a}$ & $26,4 \mathrm{a}$ & $10,7 \mathrm{a}$ & $1,4 \mathrm{~b}$ & $0,33 \mathrm{a}$ & & $28,0 \mathrm{~b}$ & $4,3 \mathrm{a}$ & $3,71 \mathrm{~b}$ & $0,43 \mathrm{~b}$ & $13,9 \mathrm{~b}$ & $31,9 \mathrm{~b}$ \\
\hline G23 & $22,7 \mathrm{~b}$ & $23,1 \mathrm{~b}$ & $7,3 \mathrm{c}$ & $1,0 \mathrm{c}$ & $0,29 \mathrm{a}$ & & $27,0 \mathrm{~b}$ & $4,1 \mathrm{~b}$ & $3,80 \mathrm{~b}$ & $0,42 \mathrm{~b}$ & $13,4 b$ & $31,7 \mathrm{~b}$ \\
\hline G24 & $24,4 \mathrm{~b}$ & $24,7 \mathrm{~b}$ & $9,6 \mathrm{~b}$ & $1,4 \mathrm{~b}$ & $0,17 \mathrm{~b}$ & 69 & $27,7 \mathrm{~b}$ & $2,5 b$ & $3,93 \mathrm{a}$ & $0,38 \mathrm{~b}$ & $13,6 b$ & $35,5 \mathrm{~b}$ \\
\hline G25 & $25,4 \mathrm{a}$ & $24,0 \mathrm{~b}$ & $10,6 \mathrm{a}$ & $1,4 \mathrm{~b}$ & $0,21 \mathrm{~b}$ & 71 & $25,6 \mathrm{~b}$ & $2,8 \mathrm{~b}$ & $3,70 \mathrm{~b}$ & $0,42 \mathrm{~b}$ & $14,4 \mathrm{~b}$ & $34,1 \mathrm{~b}$ \\
\hline G26 & $23,5 \mathrm{~b}$ & $23,1 \mathrm{~b}$ & $8,0 \mathrm{~b}$ & $1,1 \mathrm{~b}$ & $0,36 \mathrm{a}$ & 68 & $26,4 \mathrm{~b}$ & $5,0 \mathrm{a}$ & $3,74 \mathrm{~b}$ & $0,41 \mathrm{~b}$ & $12,5 \mathrm{~b}$ & $31,0 \mathrm{~b}$ \\
\hline G27 & $22,5 \mathrm{c}$ & $22,8 \mathrm{~b}$ & $7,8 \mathrm{~b}$ & $1,1 \mathrm{~b}$ & $0,30 \mathrm{a}$ & 73 & $22,4 \mathrm{c}$ & $4,2 \mathrm{a}$ & $3,68 \mathrm{~b}$ & $0,44 \mathrm{~b}$ & $13,0 \mathrm{~b}$ & $29,5 \mathrm{~b}$ \\
\hline G28 & $22,7 \mathrm{~b}$ & $23,2 \mathrm{~b}$ & $7,4 \mathrm{c}$ & $1,2 \mathrm{~b}$ & $0,21 \mathrm{~b}$ & 63 & $32,9 \mathrm{~b}$ & $3,4 \mathrm{~b}$ & $3,41 \mathrm{c}$ & $0,55 \mathrm{a}$ & $13,8 \mathrm{~b}$ & $25,3 \mathrm{c}$ \\
\hline G29 & $21,6 \mathrm{c}$ & $22,0 \mathrm{c}$ & $6,5 \mathrm{c}$ & $1,4 \mathrm{~b}$ & $0,11 \mathrm{c}$ & $64,1 \mathrm{~b}$ & $33,5 \mathrm{a}$ & $2,4 \mathrm{c}$ & $3,44 \mathrm{c}$ & $0,53 \mathrm{a}$ & $13,5 b$ & $25,7 \mathrm{~b}$ \\
\hline G30 & $22,7 \mathrm{~b}$ & $22,8 \mathrm{~b}$ & $7,6 \mathrm{~b}$ & $1,4 \mathrm{~b}$ & $0,21 \mathrm{~b}$ & $65,5 \mathrm{~b}$ & $30,7 \mathrm{~b}$ & $3,8 \mathrm{~b}$ & $3,33 \mathrm{c}$ & $0,59 \mathrm{a}$ & $12,3 \mathrm{~b}$ & $20,9 \mathrm{c}$ \\
\hline G31 & $25,9 \mathrm{a}$ & $26,5 \mathrm{a}$ & $11,0 \mathrm{a}$ & $1,6 \mathrm{a}$ & $0,20 \mathrm{~b}$ & $66,0 \mathrm{~b}$ & $31,1 \mathrm{~b}$ & $2,9 \mathrm{~b}$ & $3,52 \mathrm{~b}$ & $0,50 \mathrm{~b}$ & $14,1 \mathrm{~b}$ & $28,5 \mathrm{~b}$ \\
\hline G32 & $22,1 \mathrm{c}$ & $22,3 \mathrm{c}$ & $7,1 \mathrm{c}$ & $1,7 \mathrm{a}$ & $0,12 \mathrm{c}$ & $61,4 \mathrm{c}$ & $35,9 \mathrm{a}$ & $2,7 \mathrm{~b}$ & $3,87 \mathrm{~b}$ & $0,39 \mathrm{~b}$ & $10,8 \mathrm{c}$ & $28,9 \mathrm{~b}$ \\
\hline G33 & $24,0 \mathrm{~b}$ & $24,6 \mathrm{~b}$ & $9,1 \mathrm{~b}$ & $1,3 \mathrm{~b}$ & $0,23 \mathrm{~b}$ & $67,4 \mathrm{~b}$ & $29,6 \mathrm{~b}$ & $3,1 \mathrm{~b}$ & $3,49 \mathrm{~b}$ & $0,51 \mathrm{~b}$ & $15,3 \mathrm{a}$ & $30,6 \mathrm{~b}$ \\
\hline G34 & $22,1 \mathrm{c}$ & $22,5 \mathrm{c}$ & $8,9 \mathrm{~b}$ & $1,2 \mathrm{~b}$ & $0,24 \mathrm{~b}$ & $65,8 \mathrm{~b}$ & $31,0 \mathrm{~b}$ & $3,2 \mathrm{~b}$ & $3,37 \mathrm{c}$ & $0,53 \mathrm{a}$ & $14,4 \mathrm{~b}$ & $27,5 \mathrm{~b}$ \\
\hline G35 & $26,0 \mathrm{a}$ & $26,3 \mathrm{a}$ & $11,4 \mathrm{a}$ & $1,5 \mathrm{~b}$ & $0,16 \mathrm{~b}$ & $68,1 \mathrm{~b}$ & $29,7 \mathrm{~b}$ & $2,2 \mathrm{c}$ & $3,87 \mathrm{~b}$ & $0,37 \mathrm{c}$ & $13,5 \mathrm{~b}$ & $37,0 \mathrm{a}$ \\
\hline G36 & $23,8 \mathrm{~b}$ & $24,2 \mathrm{~b}$ & $8,6 \mathrm{~b}$ & $1,0 \mathrm{c}$ & $0,29 \mathrm{a}$ & $66,9 \mathrm{~b}$ & $29,6 \mathrm{~b}$ & $3,5 \mathrm{~b}$ & $4,01 \mathrm{a}$ & $0,35 \mathrm{c}$ & $13,8 \mathrm{~b}$ & $38,8 \mathrm{a}$ \\
\hline G37 & $24,6 \mathrm{~b}$ & $25,2 \mathrm{~b}$ & $9,4 \mathrm{~b}$ & $1,4 \mathrm{~b}$ & $0,31 \mathrm{a}$ & $66,0 \mathrm{~b}$ & $29,6 \mathrm{~b}$ & $4,4 \mathrm{a}$ & $3,50 \mathrm{~b}$ & $0,47 \mathrm{~b}$ & $14,2 \mathrm{~b}$ & $30,2 \mathrm{~b}$ \\
\hline G38 & $22,8 \mathrm{~b}$ & $23,3 \mathrm{~b}$ & $7,7 \mathrm{~b}$ & $1,4 \mathrm{~b}$ & $0,18 \mathrm{~b}$ & $67,3 \mathrm{~b}$ & $29,5 \mathrm{~b}$ & $3,2 \mathrm{~b}$ & $3,43 \mathrm{c}$ & $0,53 \mathrm{a}$ & $14,0 \mathrm{~b}$ & $26,5 \mathrm{~b}$ \\
\hline G39 & $21,8 \mathrm{c}$ & $21,7 \mathrm{c}$ & $6,4 \mathrm{c}$ & $1,3 \mathrm{~b}$ & $0,16 \mathrm{~b}$ & $61,4 \mathrm{c}$ & $35,4 \mathrm{a}$ & $3,2 \mathrm{~b}$ & $3,41 \mathrm{c}$ & $0,40 \mathrm{~b}$ & $12,2 \mathrm{~b}$ & $30,8 \mathrm{~b}$ \\
\hline G40 & $23,8 \mathrm{~b}$ & $23,9 \mathrm{~b}$ & $8,4 \mathrm{~b}$ & $1,5 \mathrm{~b}$ & $0,21 \mathrm{~b}$ & $62,6 \mathrm{c}$ & $33,7 \mathrm{a}$ & $3,7 \mathrm{~b}$ & $4,04 \mathrm{a}$ & $0,34 \mathrm{c}$ & $12,4 \mathrm{~b}$ & $36,0 \mathrm{~b}$ \\
\hline Média & 23,8 & 24,1 & 8,7 & 1,3 & 0,22 & 67,9 & 28,9 & 3,3 & 3,68 & 0,44 & 13,4 & 30,9 \\
\hline $\begin{array}{l}\text { Desvio } \\
\text { padrão }\end{array}$ & 1,21 & 1,23 & 1,29 & 0,18 & 0,06 & 4,35 & 4,13 & 0,88 & 0,23 & 0,07 & 1,19 & 5,52 \\
\hline C.V. $(\%)$ & & & & 14,29 & 26,64 & 6,41 & 14,31 & 26,74 & 6,20 & 15,03 & 8,94 & 17,85 \\
\hline
\end{tabular}

Genótipos com médias seguidas de mesma letra na coluna pertencem ao mesmo grupo, considerando um desvio padrão da média. DLF $=$ diâmetro longitudinal do fruto; $\mathrm{DTF}=$ diâmetro transversal do fruto; MFF = massa fresca do fruto; NS = número de sementes; MFS $=$ massa fresca da semente; $\mathrm{pH}=$ potencial hidrogeniônico; $\mathrm{SST}=$ sólidos solúveis totais; $\mathrm{ATT}=$ acidez total titulável. 
TABELA 2 - Coeficiente de correlação de Pearson entre características físico-químicas dos frutos de 40 genótipos de jabuticabeira (Plinia cauliflora) em um sítio de ocorrência natural. Passo Fundo, RS, 2012.

\begin{tabular}{lccccccccccc}
\hline & DTF & MFF & NS & MFS & P (\%) & C (\%) & S (\%) & pH & ATT & SST & SST/ATT \\
\cline { 2 - 12 } DLF & $0,94^{*}$ & $0,93^{*}$ & 0,19 & 0,13 & $0,44^{*}$ & $-0,42^{*}$ & $-0,21$ & 0,20 & $-0,17$ & $0,43^{*}$ & 0,32 \\
DTF & & $0,87^{*}$ & 0,17 & 0,10 & $0,41^{*}$ & $-0,38$ & $-0,23$ & 0,15 & $-0,09$ & $0,43^{*}$ & 0,27 \\
MFF & & & 0,28 & 0,03 & $0,44^{*}$ & $-0,40$ & 0,31 & 0,18 & $-0,14$ & $0,57^{*}$ & 0,36 \\
NS & & & & $-0,38$ & $0,41^{*}$ & $0,44^{*}$ & $-0,03$ & $-0,05$ & 0,04 & $-0,10$ & $-0,09$ \\
MFS & & & & & 0,10 & $-0,27$ & $0,81^{*}$ & $-0,14$ & 0,14 & $-0,20$ & $-0,26$ \\
P (\%) & & & & & & $-0,98^{*}$ & $-0,34$ & 0,08 & $-0,03$ & 0,39 & 0,18 \\
C (\%) & & & & & & & 0,15 & $-0,02$ & $-0,02$ & $-0,30$ & $-0,08$ \\
S (\%) & & & & & & & $-0,26$ & 0,24 & $-0,54^{*}$ & $-0,49^{*}$ \\
pH & & & & & & & & & $-0,93^{*}$ & 0,17 & $0,86^{*}$ \\
ATT & & & & & & & & & & $-0,10$ & $-0,87^{*}$ \\
SST & & & & & & & & & & & $0,56^{*}$ \\
\hline
\end{tabular}

*Significativo pelo teste $t(\mathrm{P} \leq 0,01) . \mathrm{DLF}=$ diâmetro longitudinal do fruto; $\mathrm{DTF}=$ diâmetro transversal do fruto; $\mathrm{MFF}=$ massa fresca do fruto; $\mathrm{NS}=$ número de sementes; $\mathrm{MFS}=$ massa fresca da semente; $\mathrm{P}(\%)=$ porcentagem de polpa; $\mathrm{C}(\%)=$ porcentagem de casca; $\mathrm{S}(\%)=$ porcentagem de semente; $\mathrm{pH}=$ potencial hidrogeniônico; $\mathrm{SST}=$ sólidos solúveis totais; ATT = acidez total titulável.

TABELA 3 -Contribuição relativa dos caracteres para a divergência genética (CRDG) (SINGH, 1981) entre 40 genótipos de jabuticabeira (Plinia cauliflora) em um sítio de ocorrência natural. Passo Fundo, RS, 2012.

\begin{tabular}{lrc}
\hline Caractere* $^{*}$ & CRDG & Valor (\%) \\
\hline DLF & $2.288,23$ & 1,999 \\
DTF & $2.350,91$ & 2,054 \\
MFF & $2.597,28$ & 2,269 \\
NS & 53,32 & 0,047 \\
MFS & 5,46 & 0,005 \\
Polpa (\%) & $29.484,02$ & 25,759 \\
Casca (\%) & $26.602,05$ & 23,241 \\
Semente (\%) & $1.195,04$ & 1,044 \\
pH & 81,07 & 0,071 \\
ATT & 6,94 & 0,006 \\
SST & $2.222,07$ & 1,941 \\
SST/ATT & $47.575,75$ & 41,565 \\
\hline
\end{tabular}

*DLF = diâmetro longitudinal do fruto; $\mathrm{DTF}=$ diâmetro transversal do fruto; $\mathrm{MFF}=$ massa fresca do fruto; NS = número de sementes; MFS = massa fresca da semente; Polpa $(\%)=$ porcentagem de polpa; Casca $(\%)=$ porcentagem de casca; Semente $(\%)=$ porcentagem de semente; $\mathrm{pH}=$ potencial hidrogeniônico; $\mathrm{SST}$ = sólidos solúveis totais; ATT = acidez total titulável. 


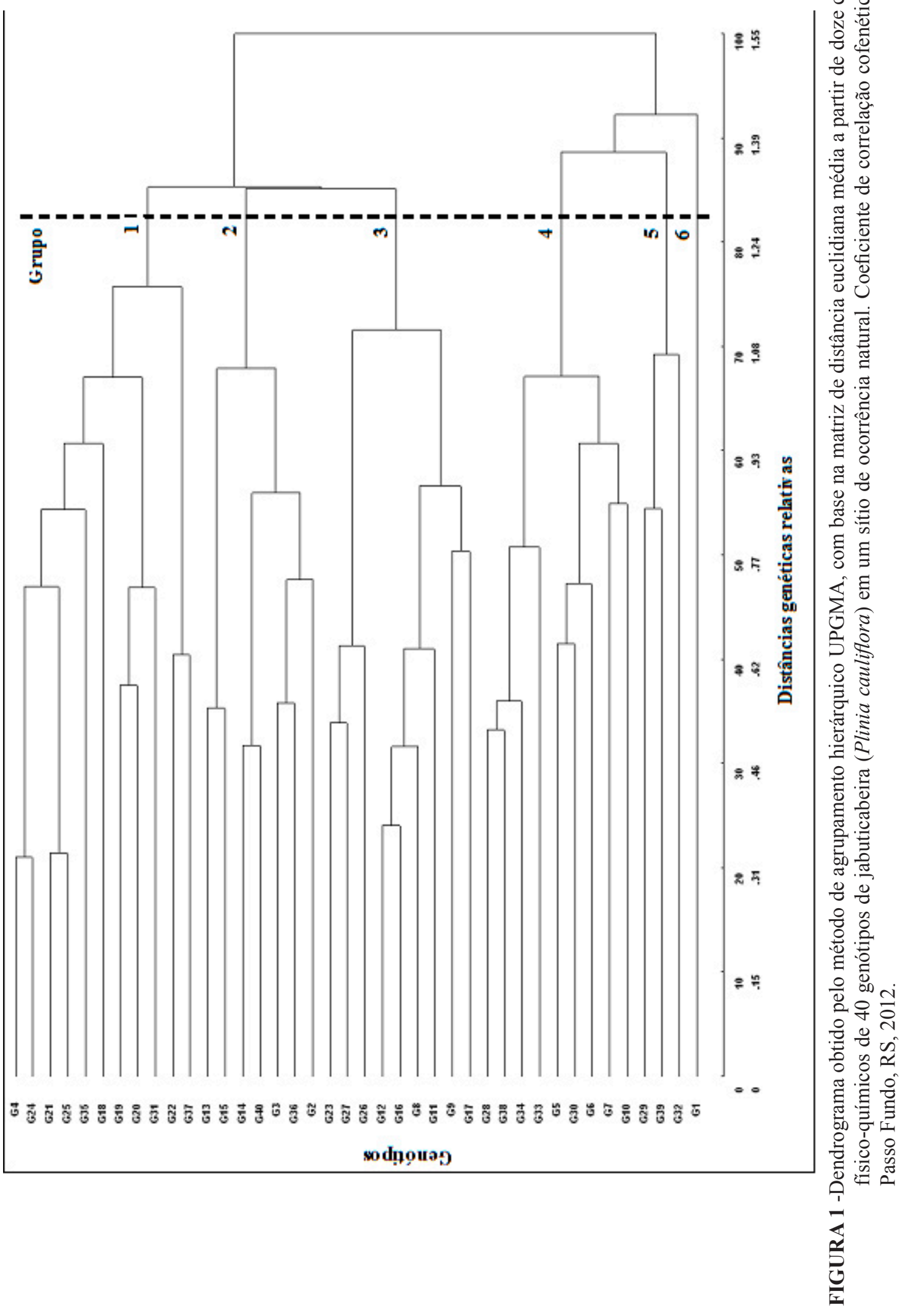




\section{CONCLUSÃO}

Jabuticabeiras da espécie Plinia cauliflora, nativas de um mesmo sítio de ocorrência, apresentam frutos com características físico-químicas variáveis, revelando a existência de genótipos com caracteres de interesse no melhoramento da espécie.

\section{AGRADECIMENTOS}

Ao CNPq e FAPERGS, pelo apoio financeiro concedido através do Edital 008/2009 Pronex, e Bolsa Produtividade CNPq concedida ao segundo autor deste artigo.

\section{REFERÊNCIAS}

CARVALHO, L.P. de; LANZA, M.A.; FALLIERI, J.; SANTOS, J.W. Análise da diversidade genética entre acessos de banco ativo de germoplasma de algodão. Pesquisa Agropecuária Brasileira, Brasília, v.38, n.10, p.1149-1155, 2003.

CITADIN, I.; DANNER, M.A.; SASSO, S.A.Z. Jabuticabeiras. Revista Brasileira de Fruticultura, Jaboticabal, v.32, n.2, p.577-583, 2010.

CITADIN, I.; VICARI, I.J.; SILVA, T.T.; DANNER, M.A. Qualidade de frutos de jabuticabeira (Myrciaria cauliflora) sob influência de duas condições de cultivo: sombreamento natural e pleno sol. Revista Brasileira de Agrociência, Pelotas, v.11, n.3, p.373375, 2005.

CRUZ, C.D. Programa GENES: análise multivariada e simulação. Viçosa: UFV, 2006. 175p.

CRUZ, C.D.; REGAZZI, A.J. Modelos biométricos aplicados ao melhoramento genético. 2.ed. Viçosa: UFV, 1997. 390 p.

DANNER, M.A.; CITADIN, I.; FERNANDES JUNIOR, A.A.; ASSMANN, F.; MAZARO, S.M.; DONAZZOLO, J.; SASSO, S.A.Z. Enraizamento de Jabutacabeira (Plinia trunciflora) por mergulhia aérea. Revista Brasileira de Fruticultura, Jaboticabal, v.28, n.3, p.530-532, 2006.

DANNER, M.A.; CITADIN, I.; SASSO, S.A.Z.; SCARIOT, S.; BENIN, G. Genetic dissimilarity among jabuticaba trees native to Southwestern Paraná, Brazil. Revista Brasileira de Fruticultura, Jaboticabal, v.33, n.2, p.517-525, 2011.
DANNER, M.A.; CITADIN, I.; SASSO, S.A.Z.; TOMAZONI, J.C. Diagnóstico ecogeográfico da ocorrência de jabuticabeiras nativas no Sudoeste do Paraná. Revista Brasileira de Fruticultura, Jaboticabal, v.32, n.3, p.746-753, 2010.

ELIAS, H.T.; VIDIGAL, M.C.G.; GONELA, A.; VOGT, G.A. Variabilidade genética em germoplasma tradicional de feijão-preto em Santa Catarina. Pesquisa Agropecuária Brasileira, Brasília, v.42, n.10, p.1443-1449, 2007.

EMBRAPA. Centro Nacional de Pesquisa de Trigo. Laboratório de Agrometeorologia. Normais climatológicas (1961-1990). Passo Fundo, 2013. Disponível em: <http://www.cnpt.embrapa.br/ pesquisa/agromet/app/principal/normais.php $>$. Acesso em: 5 jan. 2013.

FACHINELLO, J.C.; NACHTIGAL, J.C. Colheita e armazenamento. In: NACHTIGAL, J.C.; FACHINELLO, J.C.; KERSTEN, E. Fruticultura: fundamentos e prática. Pelotas: Embrapa Clima Temperado, [s.d.]. Livro Eletrônico. Disponível em: <http://www.cpact. embrapa.br/publicacoes/ download/livro/fruticultura fundamentos pratica/12.2.htm>. Acesso em: 18 fev. 2013.

INSTITUTO ADOLFO LUTZ. Normas analíticas, métodos químicos e físicos para análise de alimentos. São Paulo: IAL, 1985. v.1, 371p.

JESUS, N.; MARTINS, A.B.G.; ALMEIDA, E.J. de; LEITE, J.B.V.; GANGA, R.M.D.; SCALOPPI JUNIOR, E.J.; ANDRADE, R.A. de; MOREIRA, R.F.C. Caracterização de quatro grupos de jabuticabeira, nas condições de Jaboticabal-SP. Revista Brasileira de Fruticultura, Jaboticabal, v.26, n.3, p.482-485, 2004.

KUINCHTNER, A.; BURIOL, G.A. Clima do estado do Rio Grande do Sul segundo a classificação climática de Köppen e Thornthwaite. Disciplinarum Scientia, Santa Maria, v.2, n.1, p.171-182, 2001.

LEITE-LEGATTI, A.V.; BATISTA, A.G.; DRAGANO, N.R.V.; MARQUES, A.C.; MALTA, L.G.; RICCIO, M.F.; EBERLIN, M.N.; MACHADO, A.R.T.; CARVALHO-SILVA, L.B.; RUIZ, A.L.T.G.; CARVALHO, J.E.; PASTORE, G.M.; MARÓSTICA JÚNIOR, M.R. Jaboticaba peel: Antioxidant compounds, antiproliferative and antimutagenic activities. Food Research International, New York, v.49, p.596-603, 2012. 
LENQUISTE, S.A.; BATISTA, A.G.; MARINELI, R.S.; DRAGANO, N.R.V.; MARÓSTICA JÚNIOR, M.R. Freeze-dried jaboticaba peel added to highfat diet increases HDL-cholesterol and improves insulin resistance in obese rats. Food Research International, New York, v.49, p.153-160, 2012.

LIMA, A. de J.B.; CORRÊA, A.D.; ALVES, A.P.C.; ABREU, C.M.P.; DANTAS-BARROS, A.M. Caracterização química do fruto jabuticaba (Myrciaria cauliflora Berg) e de suas frações. Archivos Latinoamericanos de Nutricion, Caracas, v.58, n.4, p.416-421, 2008.

MATTOS, J.R. Fruteiras nativas do Brasil: jaboticabeiras. Porto Alegre: Nobel, 1983. 92 p.
OLIVEIRA, A.L. de; BRUNINI, M.A.; SALANDINI, C.A.R.; BAZZO, F.R. Caracterização tecnológica de jabuticabas 'sabará' provenientes de diferentes regiões de cultivo. Revista Brasileira de Fruticultura, Jaboticabal, v.25, n.3, p.397-400, 2003.

STRECK, E.V.; KÄMPF, N.; DALMOLIN, R.S; D.; KLAMT, E.; NASCIMENTO, P.C. do; SCHENEIDER, P.; GIASSON, E.; PINTO, L.F.S. Solos do Rio Grande do Sul. 2. ed. Porto Alegre: Emater/RS-Ascar, 2008. 222 p.

VIEIRA, J.V.; SILVA, G.O.; BOITEUX, L.S.; SIMON, P.W. Divergência genética entre acessos de cenoura pertencentes a grupos varietais distintos utilizando caracteres morfológicos. Horticultura Brasileira, Brasília, v.27, n.4, p.473-477, 2009. 